\title{
The Influence of Pregnancy Stress, Anxiety, and Sense of Mastery on Family Function in High-Risk Pregnant Women
}

\author{
Sunghee Lee ${ }^{1}$ and Eunyoung Lee ${ }^{2 *}$ \\ ${ }^{1}$ Professor, College of Nursing, The Research Institute of Nursing, Kyungpook National \\ University, 680, Gukchaebosang-ro, Jung-gu, Daegu 41944, Korea \\ ${ }^{2 *}$ Doctoral Student, Graduate School of Nursing, Kyungpook National University,680, \\ Gukchaebosang-ro, Jung-gu, Daegu 41944, Korea \\ ${ }^{1}$ leesh@knu.ac.kr, ${ }^{2 *}$ Corresponding Author: besteun918@naver.com
}

\begin{abstract}
The present study aimed to identify factors that influence family function among high-risk pregnancy women. The study subjects included 91 high-risk pregnant women who were diagnosed with high-risk pregnancy during 20 38 weeks of gestation in three university hospitals in B, D, Y cities between March 3, 2015 and March 30, 2015, and visited obstetrics and gynecology outpatient clinics for prenatal examinations or entered delivery rooms. Collected data were analyzed by using IBM SPSS Statistics 22 software, mean and standard deviation, and tested using t-test, one-way ANOVA, and Pearson's correlation coefficients. A multiple regression analysis was performed to identify factors that influence family function among the participants. The study result showed that pregnancy stress $(r=-.556, p<.001)$ and anxiety $(r=-.576, p<.001)$ were significantly negatively correlated with family function while sense of mastery $(r=.550, p<.001)$ was significantly positively correlated with family function. Anxiety $(\beta=-.312, p=.007)$, sense of mastery $(\beta=.259, p=.010)$, gestational age $(\beta=-.255$, $p=.002)$, and pregnancy stress $(\beta=-.216, p=.037)$ were identified as factors that influence family function, and they explained $47.4 \%$ of the entire model. This study suggests the need for development and application of nursing intervention programs that can reduce pregnancy stress and anxiety and increase sense of mastery in pregnant women by providing prenatal care, and thereby improve family function among high-risk pregnant women.
\end{abstract}

Keywords: Anxiety, Family function, High-risk pregnancy, Pregnancy stress, Sense of mastery

\section{Introduction}

\subsection{Background}

The age at first marriage has been increasing among men and women, and so has been the number of women who are becoming pregnant at older ages in Korea. Accordingly, the rate of high-risk pregnancy among women has also increased. According to the Health Insurance Review and Assessment Service, while the rate of high-risk pregnancy was only $7 \%$ among women aged 25 - 30 years, the rate drastically increased to $24 \%$ among women aged 35 - 40 years, and $39 \%$ among women aged 40 years or older. $42.8 \%$ of all pregnancy cases were high-risk pregnancy cases.

Article history:

Received (August 11, 2016), Review Result (September 15, 2016), Accepted (October 19, 2016) 
High-risk pregnancy refers to a pregnancy in which conditions such as which premature labor pain, cervical incompetence, preterm premature rupture of membranes, pregnancy induced hypertension, infections, and placenta previa may endanger the health of the mother and fetus, which can only be alleviated through intensive treatment [1]. Women with highrisk pregnancy are highly anxious due to concerns for the health of their fetuses and their own, physical discomfort, and uncertainty of delivery outcomes during the pregnancy period [2]. This reduces their level of maternal confidence and negatively affects their family function [3].

Family function is defined as activities done for harmony between persons and the environment that enable the family to maintain itself as an open system [4]. Better family function means tighter family ties, and an increased ability of the family to perform its role and manage dangerous or stressful situations [5]. Particularly, situations that threaten the health of pregnant mothers and their fetuses (i.e. high-risk pregnancy) may affect family function among these pregnant women [6].

High-risk pregnant women experience a higher level of stress than normal pregnant women [3]. Pregnancy stress experienced by high-risk pregnant women negatively affects the relationships and communication among family members [7], weakening family function among high-risk pregnant women. High-risk pregnant women have a higher level of anxiety than normal pregnant women, and the level increases as the pregnancy conditions worsen [8]. A high level of anxiety threatens the health of high-risk pregnant women and their fetuses [9], induces more controlled and pessimistic behavior in these women compared to normal pregnant women, reduces the sense of closeness among family members [10], and consequently, compromises family function among high-risk pregnant women.

Sense of mastery represents a belief that one can lead a life in which he/she can bring about any changes he/she wants within the given environment as long as he/she has a will and makes good effort [11]. As can be seen, a sense of mastery is an important contributor of the psychological well-being of high-risk pregnant women [12]. Improving the sense of mastery among high-risk pregnant women will reduce their anxiety and pregnancy stress, and improve their family function.

However, domestic research on factors that influence family function of high-risk pregnant women is still lacking. Therefore, the present study aimed to investigate the influence of pregnancy stress, anxiety and sense of mastery on family function among high-risk pregnant women, and provide basic data for the development of nursing intervention programs aimed at improving family function of high-risk pregnant women.

\subsection{Purpose of the study}

The purpose of this study was to identify factors that influence family function among high-risk pregnancy women.

\section{Methods}

\subsection{Study design}

The present descriptive study investigates the influence of pregnancy stress, anxiety, and sense of mastery on family function among high-risk pregnant women. 


\subsection{Participants and data collection}

The study participants included 91 high-risk pregnant women who visited obstetrics and gynecology outpatient clinics for prenatal examinations or entered in a delivery room during 20 - 38 weeks of gestation in one of the three university hospitals located in B, D, Y cities between March 3, 2015 and March 30, 2015. The sample number was calculated using $\mathrm{G}^{*}$ power 3.1. It was calculated that a sample size of 85 would have $80 \%$ statistical power to detect differences at the $\mathrm{p}<0.05$ level of significance with an effect size of 0.15 or the four predictor variables. 120 participants were sampled to allow for a $20 \%$ nonresponse rate. Data collection was done upon the consent of the heads of the three university hospitals and with the cooperation of obstetrics and gynecology clinics and delivery rooms. Researchers conducted the survey themselves. Each questionnaire took about 10 minutes to complete. 29 insufficiently answered questionnaires were excluded. 91 completed questionnaires were used in the statistical analysis.

\subsection{Instruments}

\subsubsection{Pregnancy stress}

The instrument developed by Ahn [13] for primigravidas, and revised and complemented by Jo and Kim [16] was used to measure pregnancy stress. This instrument included 27 questions rated on a five point Likert scale. The score for the questions ranged from one point ("never been stressed") to five points ("always been stressed"). The total scores ranged from 27 to 135 points. Higher scores indicated greater pregnancy stress. The reliability of the instrument was Cronbach's $\alpha=.85$ at the time of the revision by Jo and Kim [14], and Cronbach's $\alpha=.89$ in this study.

\subsubsection{Anxiety}

The State Trait Anxiety Inventory developed by Spielberger [15] and adapted by Kim and Shin [16] was used to measure anxiety. This instrument included 20 questions rated on a 4 point Likert scale. The score for each question ranged from one point ('very much') to four points ('not at all'). The total scores ranged from 20 to 84 points. Negative questions were reverse-coded. Higher scores indicated greater anxiety. The reliability of the instrument was Cronbach's $\alpha=.92$ at the time of its adaptation by Kim and Shin [16] and Cronbach's $\alpha=.91$ in this study.

\subsubsection{Sense of mastery}

The Sense of Mastery-measuring Instrument developed by Pearlin et al. [11] and adapted by Korean Gerontology was used to measure sense of mastery. This instrument included 7 questions rated on a four point scale. The score for each question ranged from 1 point ('strongly agree') to four points ('not agree at all'). The total scores ranged from 7 to 28 . Negative questions were reverse-coded. Higher scores indicated a greater sense of mastery. The reliability of the instrument was Cronbach's $\alpha=.70$ at the time of its adaptation by Korean Gerontology, and Cronbach's $\alpha=.74$ in this study.

\subsubsection{Family function}

Feetham Family Functioning Survey (FFFS) developed by Roberts and Feetham [14], and adapted and revised by Kim was used to measure family function. This instrument included 
21 questions, and each question consisted of three subdomains (current family function, expected family function, and importance of family function). Four questions that were difficult to be answered by pregnant women who did not have any children were excluded. Only the 'current family function' subdomains were answered for each of the remaining 17 questions. Each question was in the porter Format rated on a seven point scale. The scores ranged from 1 point ('not have at all') to 7 points ('have a lot'). The total scores ranged from 21 to 147, and higher scores indicated greater family function. The reliability of the instrument was Cronbach's $\alpha=.66$ at the time of the adaptation by Kim, and Cronbach's $\alpha=$. 73 in this study.

\subsection{Data analysis}

Collected data were analyzed using the IBM SPSS Statistics 22 software. The general and obstetric characteristics were analyzed using mean and standard deviation. A t-test and oneway ANOVA were used to assess differences in the level of family function according to the general characteristics. The correlations among pregnancy stress, anxiety, sense of mastery and family function were verified using Pearson's correlation coefficient. A multiple regression analysis was performed to investigate the factors that influence family function of the participants.

\section{Results}

\subsection{The influence of pregnancy stress, anxiety, and sense of mastery on family function in the subjects}

Anxiety, sense of mastery, gestational age, and pregnancy stress were identified as factors that influence family function. They explained $47.4 \%$ of the entire model $(\mathrm{F}=21.288, p<.001)$. Anxiety had the greatest influence on family function of all the factors $(\beta=-.312, p=.007)$ [Table 1].

Table 1 . The influence of pregnancy stress, anxiety, and sense of mastery on family function in the subjects $(\mathrm{N}=91)$

\begin{tabular}{|c|c|c|c|c|c|c|c|}
\hline Variables & $\mathrm{B}$ & $\beta$ & $\mathrm{t}$ & $p$ & $\mathrm{R}^{2}$ & Adj. $\mathrm{R}^{2}$ & $\mathrm{~F}(p)$ \\
\hline (Constants) & 83.951 & & 6.620 & .001 & .498 & .474 & $\begin{array}{c}21.288 \\
(<.001)\end{array}$ \\
\hline Anxiety & -.441 & -.312 & 2.777 & .007 & & & \\
\hline Sense of mastery & 1.013 & .259 & 2.624 & .010 & & & \\
\hline Gestational age $\dagger$ & -5.775 & -.255 & -3.259 & .002 & & & \\
\hline Pregnancy stress & -.184 & -.216 & -2.122 & .037 & & & \\
\hline \\
$\begin{array}{l}\text { F Dummy coded. } \\
\text { Reference: Gestational age 28-38 weeks }\end{array}$
\end{tabular}




\section{Conclusion}

The present descriptive study aimed to investigate the influence of pregnancy stress, anxiety, and sense of mastery on family function in high-risk pregnant women, and provide basic research materials useful for the development of nursing interventions. The result showed that the level of family function increased as the level of pregnancy stress and anxiety decreased, and sense of mastery increased in high-risk pregnancy women.

Based on this finding, development of nursing support strategies that reduce the level of pregnancy stress and anxiety, and enhance the sense of mastery in high-risk pregnancy women should be considered. In addition, additional research on the factors that influence family function of high-risk pregnancy women with other various factors added must be conducted. Development of nursing interventional programs that enhance family function of high-risk pregnancy women, and research to validate their efficacy are also suggested.

\section{References}

[1] J. Queenan, C. Spong, and C. Lockwood, "Management of High-Risk pregnancy: An Evidence-Based approach", Blackwell Publishing, Malden, MA, (2007)

[2] E. H. Moon, J. Y. Kim, M. K. Jeung, H. J. Son, and J. N. Oh, "Anxiety-depression and Maternal Fetal Attachment Behaviors of Pregnant Women with Preterm Labor and Normal Pregnant Women," Korean Journal of Parent-Child Health, vol.9, no.2, pp.128-139, (2006)

[3] R. T. Mercer, S. L. Ferketich, J. DeJoseph, K. May, and D. Solid, "Effect of Stress on Family Functioning During Pregnancy,” Nursing Research, vol.37, no.5, pp.268-275, (1988)

[4] C. S. Roberts and S. L. Feetham, "Assessing Family Functioning Across Three Areas of Relationships," Nursing Research, vol.31, no.4, pp.231-235, (1982)

[5] F. Mostafavirad, M. M. Anvari, F. Ansarinejad, and L.Panaghi, "Family function and social support in Iranian self-immolated women,” Burns, vol.8, no.4, pp.556-561, (2012) DOI: 10.1016/j.burns.2011.09.009

[6] R. L Goldenberg, S. P. Cliver, J. Bronstein, G. R. Cutter, W. W. Andrews, and S. T. Mennemeyer, "Bed rest in pregnancy," Obstetrics and gynecology, vol.84, no.1, (1994)

[7] E. S. Gilbert, "Manual of high risk pregnancy and delivery," Mosby, Philadelphia, (2010)

[8] R. T. Mercer and S. L. Ferketich, "Stress and social support as predictors of anxiety and depression during pregnancy," Advances in Nursing and Science, vol.10, no.2, pp.26-39, (1988) DOI: 10.1097/00012272198801000-00008

[9] A. Yarcheski, N. E. Mahon, T. J. Yarcheski, M. M. Hanks, and B. L. Cannella, "A meta-analytic study of predictors of maternal-fetal attachment," International Journal of Nursing Studies, vol.46, no.5, pp.708-715, (2009) DOI: 10.1016/j.ijnurstu.2008.10.013

[10] J. A. Maloni, J. E. Brezinski-Tomasi, and L. A. Johnson, "Antepartum bed rest: Effect upon the family," Journal of Obstetric, Gynecologic, and Neonatal Nursing, vol.30, no.2, pp.165-173, (2001) DOI: 10.1111/j.1552-6909.2001.tb01532.x

[11] L. I. Pearlin, M. A. Lieberman, E. G. Menaghan, and J. T. Mullan, "The stress process," Journal of Health and Social Behavior, vol.22, no.4, (1981)

[12] S. H. Lee and E. Y. Lee, "The relationship between pregnancy stress and anxiety in high-risk pregnant women: the mediating effect of a sense of mastery," International Journal of Bio-Science and BioTechnology, vol.8, no.1, pp.153-162, (2015) DOI: 10.14257/ijbsbt.2016.8.1.14

[13] H. L. Ahn, "An experimental study of the effects of husband's supportive behavior reinforcement education on stress relief of primigravidas," Journal of Nurses Academic Society, vol.15, no.1, (1985)

[14] Jo, J.A., Kim, and Y.H., "Mother's prenatal environment, stress during pregnancy and children's problem behaviors," J. Hum. Ecol, (2007) 
The Influence of Pregnancy Stress, Anxiety, and Sense of Mastery on Family Function in High-Risk Pregnant Women

[15] C. D. Spielberger, "State-trait anxiety inventory. The Corsini encyclopedia of psychology," John Wiley \&Sons, New York, (2010)

[16] Kim, J.T., Shin, and D.K., "A study based on the standardization of the STAI for Korea," New Medical Journals, vol.21, pp.65-75 (1978) 